\title{
Análise de um Sensor SPCE de Nanopartículas pelo Acoplamento dos Métodos Multimodal e PGF
}

\author{
André Cruz, Geisielem Seth, Yago Conceição, Nadson Souza, Tommaso Del Rosso, Victor Dmitriev e Karlo Costa
}

Resumo-Neste artigo apresentamos uma análise eletromagnética de um sensor SPCE (Surface Plasmon Coupled Emision) acoplado a um arranjo de nanopartículas Core-Shell, excitado por uma fonte óptica monocromática. Para essa análise propomos utilizar o método Multimodal acoplado ao método PGF (Periodic Green's Function), sobre os modos de Floquet, para descrever os campos na estrutura do sensor. Os resultados mostram a excitação do modo SPCE em função do ângulo de incidência da fonte monocromática, e da concentração de nanopartículas na amostra sensoriada. Por fim, verificamos que a resposta do sensor é insensível a baixas variações do ângulo de incidência do laser.

Palavras-Chave-Sensor SPCE, Plasmônica, Método Multimodal, Funções de Green.

Abstract - In this paper we present an electromagnetic analysis of a SPCE sensor (Surface Plasmon Coupled Emision) coupled to a Core-Shell nanoparticle array, excited by a optical source. For this analysis we propose to use the Multimodal method coupled with the Periodic Green's Function method, over the Floquet modes, to describe the fields in the sensor structure. The results show the excitation of the SPCE mode as a function of the source incidence angle, and the concentration of nanoparticles in the sensed sample. Finally, we found that the sensor response is insensitive to low variations in the source incidence angle.

Keywords-SPCE Sensor, Plasmonic, Multimodal Method, Green's Function.

\section{INTRODUÇÃO}

O crescente avanço na pesquisa da plasmônica têm despertado cada vez mais o interesse de grupos de pesquisas, que objetivam o desenvolvimento de novos dispositivos ópticos. Isso se deve à elevada capacidade de miniaturização desses dispositivos, e também a versatilidade em diversas aplicações de sensoriamento óptico, e análises eletromagnéticas [1,2]. A interação entre metais e ondas eletromagnéticas, no espectro óptico, produz um comportamento oscilatório coletivo no gás de elétrons livres do metal em fase oposta ao campo incidente. Tal comportamento está diretamente relacionado à componente real negativa da permissividade elétrica do metal submetido a altas frequências, resultando na formação de uma onda de superfície com características evanescentes na interface metal/dielétrico, conhecida como onda plasmônica de superfície [3].

André Cruz ${ }^{1}$, e-mail: andcruz@ufpa.br; Geisielem Seth ${ }^{1}$, e-mail: geisielen.seth@tucurui.ufpa.br; Yago Gomes ${ }^{1}$, e-mail: yagogc.ee@gmail.com; Nadson Souza ${ }^{2}$, e-mail: nadsonwelkson@unifesspa.edu.br; Tommaso Del Rosso ${ }^{3}$, e-mail: tommaso@puc-rio.br; Victor Dmitriev ${ }^{1}$, e-mail: victor@ufpa.br; Karlo Costa $^{1}$, e-mail: karlo@ufpa.br. Universidade Federal do Pará ${ }^{1}$; Universidade Federal do Sul e Sudeste do Pará ${ }^{2}$; Pontifícia Universidade Católica do Rio de Janeiro ${ }^{3}$
Devido a essas propriedades ópticas, tornou-se frequente a aplicação de metais nobres em estruturas multicamadas para o desenvolvimento de novos dispositivos baseados no fenômeno SPR (Surface Plasmon Resonance), e na produção de nanopartículas metálicas para aplicações utilizando o fenômeno LSPR (Localized Surface Plasmon Resonance) [4-6]. Os estudos dessas características físicas têm sido de grande importância no desenvolvimento de dispositivos fotônicos SPR para aplicações em fotodetecção, biosensoriamento, espectroscopia e detecção de nanopoluentes metálicos produzidos por resíduos em processos de nanofabricação [7-9].

Um sensor SPR convencional, baseado na configuração SPCE (Surface Plasmon Coupled Emission), é constituído de uma estrutura multicamadas acoplada a um canal microfluídico contendo biomoléculas e fluoróforos (moléculas fluorescentes) imobilizados sobre a estrutura do sensor, excitado por uma fonte laser externa [10-11]. O processo de sensoriamento se baseia na resposta óptica do dispositivo, proveniente das moléculas fluorescentes, em função das propriedades eletromagnéticas da amostra. Podemos destacar o uso de sensores SPR como sensores de DNA, sensores de gases, sensores de partículas e outros baseados na interrogação eletromagnética de RI (índice de refração) $[1,4,7,8]$.

A fim de melhorar a sensibilidade desses sensores, o uso de nanopartículas metálicas tem sido relatado em vários estudos [2,10,11]. Nanopartículas de ouro (AuNps), excitadas por fontes ópticas, têm seus campos próximos eficientemente amplificados pelo fenômeno de LSPR [6,11]. Fluoróforos que interagem com o campo próximo destas AuNps têm suas fluorescências amplificadas, melhorando assim a resposta óptica do sensor. Estas AuNps utilizadas em laboratório geralmente possuem uma camada dielétrica externa, sendo chamadas como Core-Shell [10].

Neste artigo apresentamos uma análise eletromagnética de um sensor SPCE acoplado a um arranjo de nanopartículas Core-Shell, excitado por uma fonte monocromática externa. Nesta abordagem, propomos uma metodologia de solução em duas etapas: Primeiramente, utilizando o método Multimodal, determinamos os campos elétrico e magnético nas camadas do sensor, considerando que não há nanopartículas na região da amostra. Em seguida, aproximamos a amostra de nanopartículas como um arranjo periódico planar de nanodipolos, produzindo um campo espalhado descrito via método da Função de Green Periódica (PGF) fora de fase (Modos de Floquet). O modelo teórico apresentado contribui de forma valiosa no processo de desenvolvimento de novos sensores, desde que já fora validado pela comparação direta com softwares comerciais. 


\section{DESCRIÇÃo FuncIONAL DO SENSOR SPCE}

A Fig. 1 mostra uma ilustração descritiva do sensor SPCE. O sensor está acoplado a um canal microfluídico contendo a amostra de multicompostos, fluoróforos e AuNps Core-Shell.

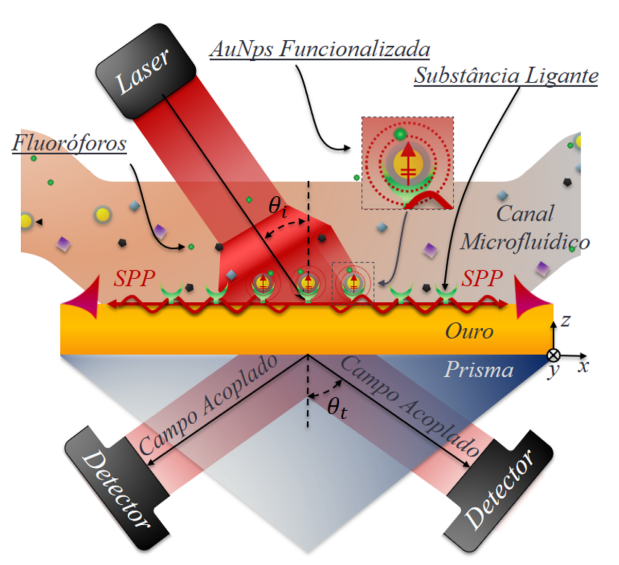

Fig. 1. Ilustração funcional do sensor SPCE acoplado à canal microfluídico.

Na região sobre a camada de ouro está presente uma substância ligante com afinidade ouro/AuNps, dessa forma, as nanopartículas ficam imobilizadas na estrutura do sensor. Esta substância eletricamente inerte atua como um espaçador químico que separa o AuNps e a camada de ouro. A excitação é realizada por um laser óptico monocromático linearmente polarizado no modo TM (Transversal Magnético) com comprimento de onda $632,8 \mathrm{~nm}$, e incide diretamente sobre a amostra com ângulo de incidência $\theta_{i}$. A radiação excita os fluoróforos funcionalmente agregados sobre as AuNps, que consequentemente re-irradiam ondas que se acoplam como modos SPP na interface, que por sua vez acoplam ondas SPCE em forma de cone na região do prisma. O cone de luz é medido por um detector e é alterado em intensidade e ângulo $\theta_{t}$ de acordo com as características eletromagnéticas da amostra $[5,8,13]$.

\section{Modelo Eletromagnético Equivalente}

No canal microfluídico a amostra possui um índice de refração efetivo definido pelos multicompostos em suspensão. $\mathrm{Na}$ amostra são inseridas AuNps Core-Shell funcionalizadas quimicamente com fluoróforos, que em seguida são capturadas pela substância ligante na superfície do sensor. A excitação do dispositivo ocorre pela incidência de uma fonte monocromática polarizada em TM, que incide com ângulo $\theta_{i}$ sobre a amostra. A incidência desse laser sob as AuNps com fluoróforos provoca a excitação do modo dipolar fundamental, e assim estas passam a se comportar como nanopartículas alvo ativas (ATN - Active target-nanoparticle), tornando-se parte do processo de transdução do sensor.

Devido a baixa concentração de ATN na superfície do sensor, podemos aproximar essa distribuição como um arranjo planar de ATN uniformemente distribuído sobre a substância ligante, a uma altura fixa da camada de ouro. Assim, a estrutura em análise é aproximada como um arranjo periódico de ATN distribuídas na região da amostra, que por sua vez está sobre a camada de ouro depositada sobre o prisma dielétrico.
A interação entre a onda plana incidente (que também é refletida na camada de ouro) e o arranjo de ATN resulta em espalhamento do campo eletromagnético.

Para determinarmos os campos EH (Elétrico e Magnético) na estrutura, é proposta uma metodologia que consiste em analisar o problema de espalhamento separadamente do problema de incidência de onda plana, identificando as soluções para ambos os problemas, e em seguida acoplando-os sendo feitas as devidas considerações. As etapas de modelagem são descritas a seguir:

- Excluir as ATN da estrutura, e então determinar a solução do problema de incidência obliquá em multicamadas pelo método Multimodal. Neste caso a estrutura do sensor é modelado como um sistema de três camadas homogêneas: Amostra/Ouro/Prisma.

- Excluir a onda plana da estrutura, e então, considerando que as nanopartículas se comportam como dipolos radiantes, determinar a solução de onda completa utilizando o método da representação espectral.

- Utilizar a equação da polarizabilidade da ATN CoreShell, para acoplar o campo elétrico da onda plana incidente (e refletida), no método Multimodal, ao momento de corrente induzido no problema periódico de espalhamento.

- Por fim, superposicionar as soluções obtidas a partir do problema de espalhamento e a partir do problema de incidência obliquá.

A solução obtida deve descrever os perfis dos campos EH espalhados na estrutura do sensor.

Primeiramente, excluindo o arranjo de nanopartículas da Fig.1, obtemos o problema de incidência oblíqua em modo TM em três meios (Fig. 2).

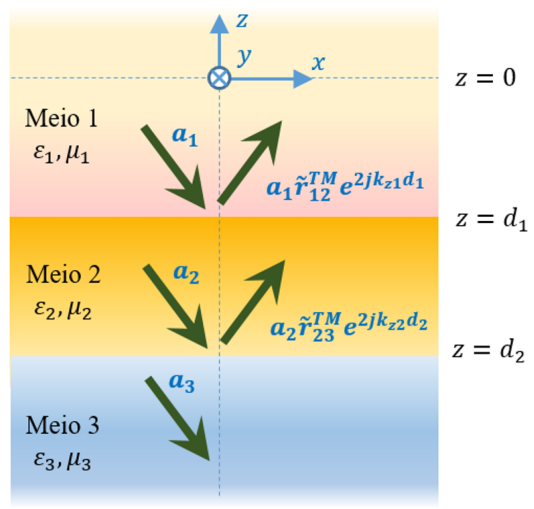

Fig. 2. Estrutura de três camadas. Método Multimodal.

Matematicamente, os campos EH das ondas TM incidente, refletidas e transmitidas podem ser descritos por exponenciais complexas que se propagam com constante de propagação $\left|\mathbf{K}_{i}\right|=\omega \sqrt{\varepsilon_{i} \mu_{i}}=\sqrt{k_{z i}^{2}+k_{x}^{2}}(\operatorname{com} i=1,2,3)$ nos três meios. Pelo método Multimodal, os campos magnéticos transversais nos três meios são dados por [14]:

$$
\mathbf{H}_{i}^{T M}(x, z)=a_{i}\left[e^{j k_{z i} z}+\tilde{r}_{i, i+1}^{T M} e^{2 j k_{z i} d_{i} e^{-j k_{z i} z}}\right] e^{-j k_{x} x}\left(-\mathbf{a}_{y}\right)
$$

onde $\tilde{r}_{i, i+1}^{T M}$ é o coeficiente de reflexão generalizado no meio $i$ definido em (2). 
Pela Lei de Ampere: $\mathbf{E}_{i}^{T M}=\left(1 / j \omega \varepsilon_{i}\right) \nabla \times \mathbf{H}_{i}^{T M}$

$$
\tilde{r}_{i, i+1}^{T M}=\frac{R_{i, i+1}^{T M}+\left[T_{i, i+1}^{T M}-R_{i, i+1}^{T M}\right] \tilde{r}_{i+1, i+2}^{T M} e^{-2 j k_{z_{i+1}}\left(d_{i}-d_{i+1}\right)}}{1+\left[T_{i, i+1}^{T M}-1\right] \tilde{r}_{i+1, i+2}^{T M} \bar{e}^{-2 j k_{z_{i+1}}\left(d_{i}-d_{i+1}\right)}}
$$

Os coeficientes de Fresnel (no modo TM) de reflexão e transmissão são, respectivamente:

$$
\begin{aligned}
R_{i, i+1}^{T M} & =\frac{\varepsilon_{i+1} k_{z_{i}}-\varepsilon_{i} k_{z_{i+1}}}{\varepsilon_{i+1} k_{z_{i}}+\varepsilon_{i} k_{z_{i+1}}} \\
T_{i, i+1}^{T M} & =\frac{2 \varepsilon_{i+1} k_{z_{i}}}{\varepsilon_{i+1} k_{z_{i}}+\varepsilon_{i} k_{z_{i+1}}}
\end{aligned}
$$

No meio $i=3$ o coeficiente generalizado $\tilde{r}_{34}^{T M}=0$, e portanto, no meio $i=2$ temos $\tilde{r}_{23}^{T M}=R_{23}^{T M}$. No meio 1 a onda incidente tem amplitude $a_{1}=H_{0} e^{-j k_{z 1} d_{1}}$ ajustada pelo campo $H_{0}$ da excitação externa. A amplitude do campo magnético transversal nos meios $i=2,3$ é definida por:

$$
a_{i}=a_{1} \frac{e^{j k_{z_{1}} d_{1}}}{e^{j k_{z_{i}} d_{i-1}}} \tilde{t}_{1 i}^{T M}
$$

onde $\tilde{t}_{1 i}^{T M}$ é o coeficiente de transmissão generalizado definido em (6)

$$
\tilde{t}_{1 i}^{T M}=\prod_{g=1}^{i-1} \frac{T_{g, g+1}^{T M} e^{-j k_{z_{g}}\left(d_{g-1}-d_{g}\right)}}{1+\left[T_{g, g+1}^{T M}-1\right] \tilde{r}_{g+1, g+2}^{T M} e^{-2 j k_{z+1}\left(d_{g}-d_{g+1}\right)}}
$$

$\mathrm{O}$ ângulo de incidência $\theta_{i}$ define a constante de propagação na direção $x$ em todos os meios, por $k_{x}=\left|\mathbf{K}_{1}\right| \sin \theta_{i}$, e assim, a constante de propagação na direção $z$ por $k_{z i}=$ $\sqrt{\omega^{2} \varepsilon_{i} \mu_{i}-k_{x}^{2}}$.

Retornando ao problema original da Fig. 1, a interação entre a onda incidente e a nanopartícula funcionalizada resulta na excitação do modo dipolar fundamental, desde que o comprimento de onda de excitação é muito maior que as dimensões da ATN. Esta interação é denominada espalhamento quase-estático de Rayleigh $[3,10]$. Portanto, desde que $\lambda \gg$ $d_{\text {eff }}$ (comprimento de onda $\lambda$ muito mair que o diâmetro efetivo da ATN $d_{e f f}$ ), o arranjo de nanopartículas pode ser aproximado como um arranjo de nanodipolos hertzianos, com polarizabilidade definida pelo tipo da nanopartícula.

Neste trabalho consideramos utilizar nanopartículas de ouro do tipo Core-Shell, muito utilizadas em aplicações de fotônica [3,10,11]. A equivalência utilizando a aproximação quase estática de Rayleigh é ilustrada na Fig. 3, cujas especificações da ATN Core-Shell são descritas.

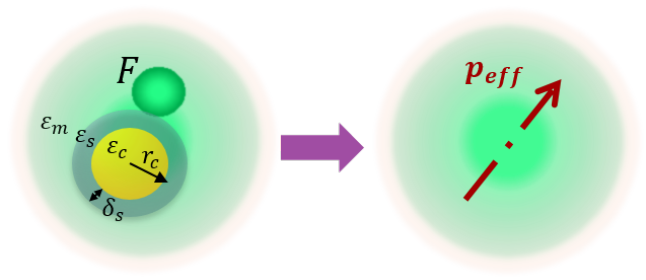

Fig. 3. Equivalente entre ATN excitada e dipolo hertziano.

Na Fig. 3, $r_{c}$ é o raio interno do núcleo da AuNps CoreShell, com permissividade $\varepsilon_{c}, \delta_{s}$ é a espessura da casca dielétrica com permissividade $\varepsilon_{s}$, e $\varepsilon_{m}$ é a permissividade do meio onde está inserida a AuNps Core-Shell.
O momento de dipolo efetivo da AuNps Core-Shell agregada ao fluoróforo (ATN equivalente) pode ser aproximado por: $\mathbf{p}_{e f f}=\left(\alpha_{C S}+\alpha_{f f} R_{e}^{f f}\right) \mathbf{E}_{1}^{T M}$, onde $\alpha_{f f}$ é a polarizabilidade do fluoróforo, $R_{e}^{f f}$ é a reação ao campo $\mathbf{E}_{1}^{T M}$ e $\alpha_{C S}$ é a polarizabilidade da AuNps Core-Shell, definida em (7) [10].

$\alpha_{C S}=4 \pi \varepsilon_{m} r_{c}^{3} \frac{1}{f}\left[\frac{f\left(\varepsilon_{c}-\varepsilon_{m}\right)\left(2 \varepsilon_{s}+\varepsilon_{m}\right)+\left(2 \varepsilon_{s}+\varepsilon_{c}\right)\left(\varepsilon_{s}-\varepsilon_{m}\right)}{f\left(\varepsilon_{c}-\varepsilon_{m}\right)\left(2 \varepsilon_{s}-2 \varepsilon_{m}\right)+\left(2 \varepsilon_{s}+\varepsilon_{c}\right)\left(\varepsilon_{s}+2 \varepsilon_{m}\right)}\right]$

sendo $f=r_{c}^{3} /\left(r_{c}+\delta_{s}\right)^{3}$ a fração do volume total da partícula ocupada pelo núcleo.

Na Fig. 4 é mostrado a arranjo periódico planar de dipolos na estrutura de três camadas.

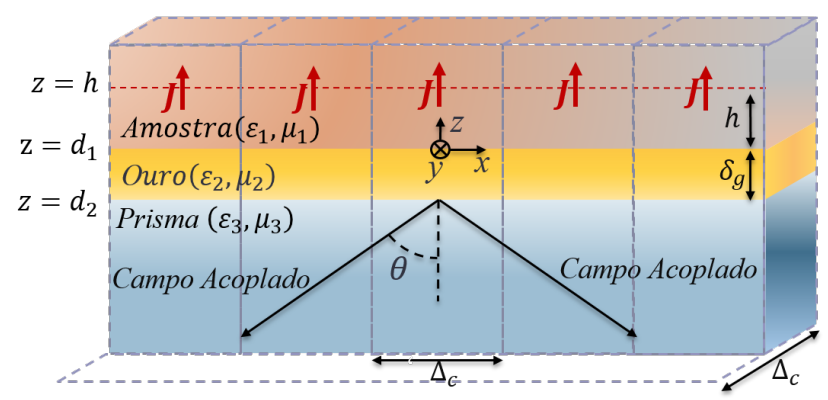

Fig. 4. Modelo equivalente: Arranjo de dipolos na estrutura de três camadas.

Devido a periodicidade bidimensional do problema, podemos determinar os campos EH pela simples escolha de uma célula de análise com largura $\Delta_{c}$ em $x$ e $y$. Apesar desta periodicidade, devemos ter em mente que a excitação do arranjo ocorre fora de fase, em função do campo elétrico $\mathbf{E}_{1}^{T M}$. Podemos verificar que o defasamento entre células ocorre com $\Delta \Psi=\Delta_{c}\left|\mathbf{K}_{1}\right| \sin \theta_{i}$. No centro da célula de análise, posicionado em $\mathbf{r}^{\prime \prime}=(0,0, h)$, o dipolo equivalente $\mathbf{J}\left(\mathbf{r}, \mathbf{r}^{\prime \prime}\right)$ tem sua orientação definida pelo campo $\mathbf{E}_{1}^{T M}(0,0, h)$, de modo que $\mathbf{J}\left(\mathbf{r}, \mathbf{r}^{\prime \prime}\right)=j \omega \mathbf{p}_{e f f}(0,0, h) \delta\left(\mathbf{r}-\mathbf{r}^{\prime \prime}\right)$.

A solução para este problema de espalhamento pode ser obtida por meio do potencial magnético definido via método da Função de Green Periódica, sobre os modos de Floquet. Este método se baseia em representar os campos EH como superposições de ondas com multiplas constantes de propagação. O formalismo completo baseado na Transformada Série de Fourier é descrito em [13]. A partir do método PGF, o potencial magnético para o arranjo planar, com período bidimensional $\Delta_{c}$, localizado em $z=h$, sobre as interfaces $z=d_{1}$ e $z=d_{2}\left(\operatorname{com} d_{1}>d_{2}\right)$, é definido em (8).

$$
\begin{array}{r}
\mathbf{A}=\sum_{m=-\infty}^{\infty} \sum_{n=-\infty}^{\infty}\left[\begin{array}{rrr}
A_{x x}^{m n} & 0 & 0 \\
0 & A_{y y}^{m n} & 0 \\
-j k_{x}^{m} A_{z x}^{m n} & -j k_{y}^{n} A_{z y}^{m n} & A_{z z}^{m n}
\end{array}\right] \frac{j \omega \mathbf{p}_{e f f}}{2 \Delta_{c}} \\
\times e^{-j\left(k_{x}^{m} x+k_{y}^{n} y\right)}
\end{array}
$$

sendo o elemento $A_{i j}$ a componente $i$, excitada pela componente $j$ da dipolo efetivo $\mathbf{p}_{\text {eff }}$ em $\mathbf{r}^{\prime \prime}=(0,0, h)$.

Em (8) $m$ e $n$ são as variáveis discretas da representação espectral, com autovalores $\left(k_{x}^{m}\right)^{2}=\left[2 m \pi / \Delta_{c}+\Delta \Psi / \Delta_{c}\right]^{2} \mathrm{e}$ $\left(k_{y}^{n}\right)^{2}=\left[2 n \pi / \Delta_{c}\right]^{2}$, devidamente associados as autofunções próprias pertinentes ao problema periódico fora de fase. 
Definimos $k_{z u}=\sqrt{\omega^{2} \varepsilon_{u} \mu_{u}-k_{x}^{m^{2}}-k_{y}^{n^{2}}}$ como a constante de propagação na direção $z$, nos meios $u=1,2,3$ com $\Im\left\{k_{z u}\right\}<0$. Os elementos do tensor potencial magnético (8), no domínio espectral $m n$ e espacial $z$, no meio 1 são:

$$
\begin{gathered}
A_{x x_{1}}^{m n}=A_{y y_{1}}^{m n}=\mu_{1} \frac{1}{j k_{z 1}}\left[e^{-j k_{z 1}|z-h|}+\tilde{R}_{12}^{T E} e^{-j k_{z 1}\left(z+h-2 d_{1}\right)}\right] \\
A_{z x_{1}}^{m n}=A_{z y_{1}}^{m n}=\mu_{1} S_{1 d 1}^{E M} \frac{1}{j k_{z 1}} e^{-j k_{z 1}\left(z+h-2 d_{1}\right)} \\
A_{z z_{1}}^{m n}=\mu_{1} \frac{1}{j k_{z 1}}\left[e^{-j k_{z 1}|z-h|}+\tilde{R}_{12}^{T M} e^{-j k_{z 1}\left(z+h-2 d_{1}\right)}\right]
\end{gathered}
$$

No meio 2:

$$
\begin{gathered}
A_{x x_{2}}^{m n}=A_{y y_{2}}^{m n}=\mu_{2} \frac{1}{j k_{z 2}} \frac{\tilde{T}_{23}^{T E}}{T_{23}^{T E}}\left[\frac{e^{j k_{z 2}\left(z-d_{2}\right)}+R_{23}^{T E} e^{-j k_{z 2}\left(z-d_{2}\right)}}{e^{j k_{z 1}\left(h-d_{1}\right)}}\right] \\
A_{z x_{2}}^{m n}=A_{z y_{2}}^{m n}=\mu_{2} \frac{1}{j k_{z 2}} \tilde{T}_{23}^{T M}\left[\frac{S_{2 d 1}^{E M} e^{j k_{z 2}\left(z-d_{2}\right)}+S_{2 d 2}^{E M} e^{-j k_{z 2}\left(z-d_{2}\right)}}{e^{j k_{z 1}\left(h-d_{1}\right)}}\right] \\
A_{z z_{2}}^{m n}=\mu_{2} \frac{1}{j k_{z 2}} \frac{\varepsilon_{2}}{\varepsilon_{1}} \frac{\tilde{T}_{23}^{T M}}{T_{23}^{T M}}\left[\frac{e^{j k_{z 2}\left(z-d_{2}\right)}+R_{23}^{T M} e^{-j k_{z 2}\left(z-d_{2}\right)}}{e^{j k_{z 1}\left(h-d_{1}\right)}}\right]
\end{gathered}
$$

No meio 3:

$$
\begin{gathered}
A_{x x_{3}}^{m n}=A_{y_{3}}^{m n}=\mu_{3}\left[\frac{\mu_{2} k_{z 3}}{\mu_{3} k_{z 2}}\right] \tilde{T}_{23}^{T E} \frac{1}{j k_{z 3}} e^{j\left[k_{z 3}\left(z-d_{2}\right)-k_{z 1}\left(h-d_{1}\right)\right]} \\
A_{z x_{3}}^{m n}=A_{z y_{3}}^{m n}=\mu_{3} S_{3 d 2}^{E M} \frac{1}{j k_{z 3}} e^{j\left[k_{z 3}\left(z-d_{2}\right)-k_{z 1}\left(h-d_{1}\right)\right]} \\
A_{z z_{3}}^{m n}=\mu_{3}\left[\frac{\varepsilon_{2} k_{z 3}}{\varepsilon_{1} k_{z 2}}\right] \tilde{T}_{23}^{T M} \frac{1}{j k_{z 3}} e^{j\left[k_{z 3}\left(z-d_{2}\right)-k_{z 1}\left(h-d_{1}\right)\right]}
\end{gathered}
$$

Os coeficientes de Fresnel dos modos TE e TM são definidos em (3) e (4). Os coeficientes generalizados de reflexão e transmissão TE e TM para o campo potencial magnético, pelo método PGF, são definidos a partir de (18) a (21).

$$
\begin{gathered}
\tilde{R}_{12}^{T E}=1-\frac{T_{21}^{T E}\left[1-R_{23}^{T E} e^{2 j k_{z 2}\left(d_{2}-d_{1}\right)}\right]}{\left[1-R_{21}^{T E} R_{23}^{T E} e^{2 j k_{z 2}\left(d_{2}-d_{1}\right)}\right]} \\
\tilde{T}_{23}^{T E}=\frac{T_{21}^{T E} T_{23}^{T E} e^{j k_{z 2}\left(d_{2}-d_{1}\right)}}{\left[1-R_{21}^{T E} R_{23}^{T E} e^{2 j k_{z 2}\left(d_{2}-d_{1}\right)}\right]} \\
\tilde{R}_{[1,3] 2}^{T M}=1-\frac{T_{2[1,3]}^{T M}\left[1-R_{2[3,1]}^{T M} e^{2 j k_{z 2}\left(d_{2}-d_{1}\right)}\right]}{\left[1-R_{21}^{T M} R_{23}^{T M} e^{2 j k_{z 2}\left(d_{2}-d_{1}\right)}\right]} \\
\tilde{T}_{23}^{T M}=\frac{T_{21}^{T M} T_{23}^{T M} e^{j k_{z 2}\left(d_{2}-d_{1}\right)}}{\left[1-R_{21}^{T M} R_{23}^{T M} e^{2 j k_{z 2}\left(d_{2}-d_{1}\right)}\right]}
\end{gathered}
$$

OS coeficientes de acoplamento $S^{T E M}$ relacionam os coeficientes de reflexão e transmissão dos modos TE e TM:

$$
\begin{aligned}
& S_{1 d 1}^{E M}=\frac{1}{2 j k_{z 2}}\left[\zeta_{12}\left[\frac{\mu_{1} \varepsilon_{1} k_{z 2}}{\mu_{2} \varepsilon_{2} k_{z 1}}\right]\left[1+\tilde{R}_{12}^{T M}\right]\left[1+\tilde{R}_{12}^{T E}\right]+\zeta_{23}\left[\frac{\mu_{2} \varepsilon_{2} k_{z 1}}{\mu_{3} \varepsilon_{3} k_{z 2}}\right] \tilde{T}_{23}^{T M} \tilde{T}_{23}^{T E}\right] \\
& S_{2 d 1}^{E M}=\frac{1}{2}\left[\left[\frac{\left[\zeta_{12} \mu_{1}\right.}{\mu_{2} k_{z 1}}\right] \frac{1}{T_{23}^{T M}}\left[1+\tilde{R}_{12}^{T E}\right]+\left[\frac{\zeta_{23} \mu_{2} \varepsilon_{2}}{\mu_{3} \varepsilon_{3} k_{z 2}}\right] \frac{R_{21}^{T M}}{T_{21}^{T M}} \tilde{T}_{23}^{T M} \tilde{T}_{23}^{T E} e^{j k_{z 2}\left(d_{2}-d_{1}\right)}\right] \\
& \left.S_{2 d 2}^{E M}=\frac{1}{2}\left[\frac{\left[\zeta_{12} \mu_{1}\right.}{\mu_{2} k_{z 1}}\right] \frac{R_{23}^{T M}}{T_{23}^{T M}}\left[1+\tilde{R}_{12}^{T E}\right]+\left[\frac{\zeta_{23} \mu_{2} \varepsilon_{2}}{\mu_{3} \varepsilon_{3} k_{z 2}}\right] \frac{1}{T_{21}^{T M}} \tilde{T}_{23}^{T M} \tilde{T}_{23}^{T E} e^{-j k_{z 2}\left(d_{2}-d_{1}\right)}\right] \\
& S_{3 d 2}^{E M}=\frac{1}{2 j k_{z 2}}\left[\zeta_{12}\left[\frac{\mu_{1} k_{z 3}}{\mu_{2} k_{z 1}}\right] \tilde{T}_{23}^{T M}\left[1+\tilde{R}_{12}^{T E}\right]+\zeta_{23}\left[\frac{\mu_{2}}{\mu_{3}}\left[1+\tilde{R}_{32}^{T M}\right] \tilde{T}_{23}^{T E}\right]\right.
\end{aligned}
$$

sendo $\zeta$ parâmetros de acoplamento nas interfaces $d_{1}$ e $d_{2}$ :

$$
\begin{aligned}
& \zeta_{12}=\left[\frac{\varepsilon_{2} \mu_{2}}{\varepsilon_{1} \mu_{1}}-1\right] \\
& \zeta_{23}=\left[\frac{\varepsilon_{3} \mu_{3}}{\varepsilon_{2} \mu_{2}}-1\right]
\end{aligned}
$$

Uma vez conhecido $\mathbf{A}$, podemos encontrar os campos $\mathbf{E}_{i}^{s c t}$ e $\mathbf{H}_{i}^{\text {sct }}$ espalhados a partir das operações diferenciais [12,13]:

$$
\begin{gathered}
\mathbf{H}_{i}^{s c t}=\frac{1}{\mu_{i}} \nabla \times \mathbf{A}_{i} \\
\mathbf{E}_{i}^{s c t}=\frac{1}{j \omega \varepsilon_{i}} \nabla \times \frac{1}{\mu_{i}} \nabla \times \mathbf{A}_{i}
\end{gathered}
$$

Por fim, utilizando o principio de superposição, os campos EH resultantes da interação entre a onda plana e o arranjo de ATN são definidos em (30) e (31).

$$
\begin{aligned}
\mathbf{E}_{i}^{\text {total }}(\mathbf{r}) & =\mathbf{E}_{i}^{T M}(\mathbf{r})+\mathbf{E}_{i}^{\text {sct }}(\mathbf{r}) \\
\mathbf{H}_{i}^{\text {total }}(\mathbf{r}) & =\mathbf{H}_{i}^{T M}(\mathbf{r})+\mathbf{H}_{i}^{\text {sct }}(\mathbf{r})
\end{aligned}
$$

\section{RESUltados}

Para verificarmos os resultados de campo através do modelo proposto, consideramos que as AuNps Core-Shell são funcionalizadas quimicamente com substâncias luminescentes, assim, adquirindo uma polarizabilidade de $\alpha_{\text {eff }}=(66,99-$ $j 3,39) D \mu m / V$. Na estrutura do sensor, o meio 1 é formado pelo arranjo planar periódico de ATN, uniformemente distribuídas a uma distância $h=20 \mathrm{~nm}$ da superfície do sensor $\left(d_{1}=0\right)$ pelo espaçador químico. A folha de ouro possui espessura de $50 \mathrm{~nm}\left(d_{2}=-50 \mathrm{~nm}\right)$.

As ATN são irradiadas por uma fonte externa de $\lambda=$ $632,8 \mathrm{~nm}$, com ângulo de incidência $\theta_{i}$. Assim, o modo dipolar fundamental é excitado na direção do campo $\mathbf{E}_{1}^{T M}(0,0, h)$, re-irradiando ondas de mesmo comprimento. A estrutura não magnética $\left(\mu_{r 1, r 2, r 3}=1\right)$ é caracterizada eletricamente, no comprimento de onda em questão, pelas permissividades do prisma (vidro óptico BK7) $\varepsilon_{r 3}=2,30$ e da camada de ouro (Modelo de Lorentz-Drude) $\varepsilon_{r 2}=-11,63-1,34 j$. A permissividade relativa da amostra é escolhida como $\varepsilon_{r 1}=1$ em um primeiro momento, para verificarmos as características de excitação SPCE independente da amostra.

Primeiramente, devemos verificar se há excitação de ondas SPCE na estrutura sem a presença do arranjo de ATN. Assim, na Fig. 5a é mostrada a amplitude $a_{3}$ (5) do campo $\mathbf{H}_{3}^{\text {total }}$ (31) na região do prisma em função do ângulo de incidência $\theta_{i}$ para $p_{e f f}=0$.
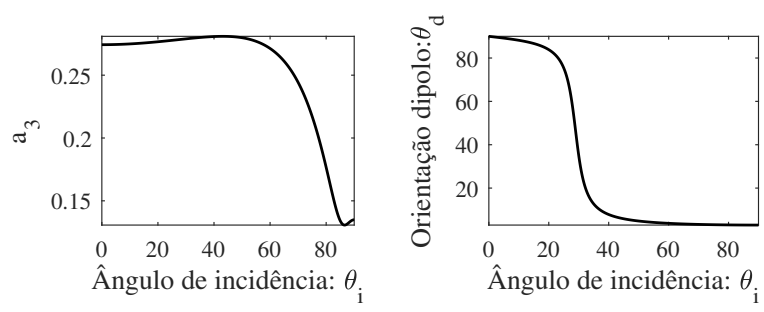

Fig. 5. (a) Amplitude do campo $\mathbf{H}_{3}^{\text {total }}$ transmitido na estrutura sem ATN (b) Orientação do arranjo de ATN em função do ângulo de incidência.

A partir da Fig. 5a podemos verificar que não há transmissão diretiva SPCE apenas com a incidência da onda TM na estrutura multicamadas. Para excitação de modos SPCE na estrutura do sensor, é necessário que os dipolos equivalentes radiem componentes capazes de acoplar ondas SPP na camada de ouro. Isto só é possível quando os dipolos são predominantemente verticais. 
Para verificar a orientação dos dipolos equivalentes em função do ângulo de incidência da onda TM, na Fig. 5b é mostrada a orientação $\theta_{d}$ do momento de dipolo $p_{\text {eff }}$ em função de $\theta_{i}$. Neste $\theta_{d}$ é o ângulo entre $p_{\text {eff }}$ e $\hat{a}_{z}$.

A partir da Fig. 5b, verificamos que para $\theta_{i}=0^{\circ}$ a excitação dipolar é horizontal $\left(\theta_{d}=90^{\circ}\right)$, e a medida que $\theta_{i}$ aumenta, $\theta_{d}$ diminui bruscamente. Para incidência com $\theta_{i}>30$ a orientação do dipolo tende a ser vertical $(\theta \approx 0)$, e desta forma será radiado componentes predominantemente em modo TM pelo arranjo, capazes de excitar modos SPP na interface amostra/ouro, acoplando assim ondas SPCE na região do Prisma.

A onda incidente e o arranjo de ATN fazem parte do processo de transdução óptica, e não devem provocar variações relevantes na sensibilidade do sensor. $\mathrm{Na}$ Fig. 6 verificamos a transmissão de $\left|A_{z z_{3}}^{m n^{\circ}}\right|$ (17) na região do prisma para três incidências: $\theta_{i}=15^{\circ}, 30^{\circ}$ e $45^{\circ}$, considerando dois arranjos de ATN com: $\Delta_{c}=5 \mu m$ e $50 \mu m$. Os resultados estão em função do ângulo de transmissão $\theta_{t}=\tan ^{-1}\left\{k_{x} / k_{z 3}\right\}$.
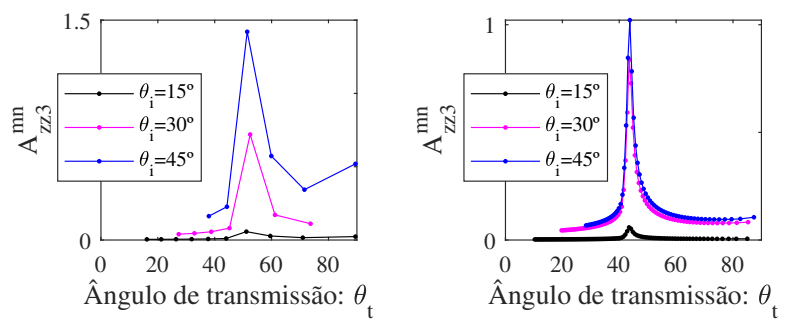

Fig. 6. Campo $\mathbf{A}_{3}$ transmitido na região do prisma para $\theta_{i}=15^{\circ}, 30^{\circ} \mathrm{e}$ $45^{\circ}$, com: (a) $\Delta_{c}=5 \mu m$, (b) $\Delta_{c}=50 \mu m$.

Na Fig. 6, como esperado, o ângulo de incidência altera a intensidade do campo transmitido, desde que controla a excitação do modo SPCE. A concentração de ATN no arranjo altera levemente o ângulo SPCE, ocorrendo próximo de $51,42^{\circ}$ para $\Delta_{c}=5 \mu \mathrm{m}$, e $43,74^{\circ}$ para $\Delta_{c}=50 \mu \mathrm{m}$. Para fins de sensoriamento, deve-se manter um controle rigoroso da concentração de AuNps e fluoróforos na amostra.

O sensor SPCE é um refratómetro, medindo as variações refrativas da amostra. Na Fig. 7 verificamos a resposta do sensor SPCE para quatro amostras $\varepsilon_{r 1}$ distintas. Nesta utilizamos uma excitação com $\theta_{i}=30^{\circ}$ e arranjo com $\Delta_{c}=50 \mu \mathrm{m}$.

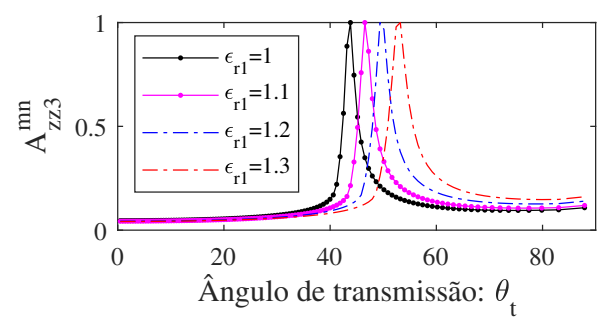

Fig. 7. Campo $\mathbf{A}_{3}$ transmitido na região do prisma para $\theta_{i}=30^{\circ}$ e $\Delta_{c}=$ $50 \mu m$, para amostras com $\varepsilon_{r 1}=1,1.1,1.2$ e 1.3 .

A partir da Fig. 7 verificamos a variação do ângulo SPCE transmitido variando com a permissividade relativa da amostra. Neste verificamos uma sensibilidade média $S=$ $\Delta \theta_{S P P} / \Delta \varepsilon_{r 1}$ de $27.85^{\circ} R I U^{-1}$.
Por fim, utilizando (31), na Fig. 8 é mostrada a componente $H_{y}$ do campo magnético na estrutura do sensor para $\theta_{i}=30^{\circ}$ aplicado ao arranjo $\Delta_{c}=5 \mu \mathrm{m}$ em uma amostra $\varepsilon_{r 1}=1.1$.

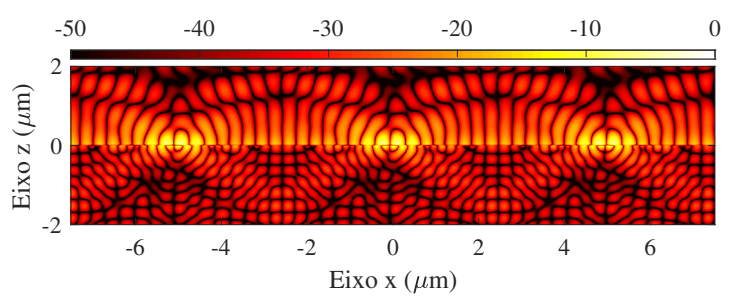

Fig. 8. Campo magnético: $20 \log \left|\Re\left\{H_{y}\right\}\right|$ em três células, com $y=0$. Séries da PGF truncadas em $m=-10: 10$ e $n=-10: 10$

\section{CONClusões}

Neste trabalho foi mostrada a análise de um sensor SPCE acoplado a um arranjo de AuNps Core-Shell. Nesta abordagem propomos descrever os campos EH na estrutura utilizando os modelos Multimodal e PGF devidamente combinados. Na Fig. 8 verificamos que todas as condições periódicas fora de fase são atendidas. Verificamos também como $\theta_{i}$ e $\Delta_{c}$ podem ser ajustados para pouco interferir na resposta do sensor. Na Fig. 7 notamos que o ângulo SPCE é alterado pela $\varepsilon_{r 1}$ da amostra, mostrando que o dispositivo opera como refratômetro.

\section{AGRADECIMENTOS}

Os autores agradecem ao apoio da Coordenação de Aperfeiçoamento de Pessoal de Nível Superior - Brasil (CAPES).

\section{REFERÊNCIAS}

[1] PERÇIN, I. et al. Microcontact imprinted plasmonic nanosensors: powerful tools in the detection of Salmonella paratyphi. Journal Sensors, v. 1,n. 6, p.1375, 2017.

[2] SZUNERITS, S. et al. Magneto-optical nanostructures for viral sensing. Nanomaterials, v. 10, n. 7, p. 1271, 2020.

[3] MAIER S. A. Plasmonics: Fundamentals and Applications, 1st ed. NewbYork, NY, USA: Springer pp. 6587, 2007.

[4] ZHENG, G. et al. High-resolution surface plasmon resonance sensor with Fano resonance in waveguide-coupled multilayer structures. Applied Physics Express, v. 10, n. 4, p. 042202, 2017.

[5] CONCEIÇÃO, Y. G. et al. Numerical Analysis of a Graphene-based SPR Sensor by the Finite Element Method. In: International Microwave and Optoelectronics Conference (IMOC), p. 1-3, 2019.

[6] MAYER, K. M. \& HAFNER, J. H. Localized surface plasmon resonance sensors. Chemical reviews, v. 111, n. 6, p. 3828-3857, 2011.

[7] WU, L. et al. Ultrasensitive biosensors based on long-range surface plasmon polariton and dielectric waveguide modes. Photonics Research, v. 4, n. 6, p. 262-266, 2016.

[8] GRYCZYNSKI, I. et al. Radiative decay engineering experimental studies of surface plasmon-coupled directional emission. Analytical biochemistry, Elsevier, v. 324, n. 2, p. 170-182, 2004.

[9] LIN, W. et al. Propagating surface plasmon polaritons for remote excitation surface-enhanced Raman scattering spectroscopy. Applied Spectroscopy Reviews, v. 53, n. 10, p. 771-782, 2018.

[10] CRUZ, A. \& COSTA, K. Extinction efficiency and electromagnetic fields of isolated and coupled core-shell nanoparticles. Journal of Communication and Information Systems, 33(1), 2018.

[11] COSTA, J. et al. Limits of the effective medium theory in particle amplified SPR spectroscopy biosensors, Sensors, 19, (3), p. 584, 2019.

[12] DUDLEY, D. G. Mathematical foundations for electromagnetic theory. New York: IEEE press, 1994.

[13] CRUZ, A. et al. Electromagnetic model of a nanodipole array above a double-layer graphene by periodic green's function. IET Microwaves, Antennas \& Propagation, 14(15), 2088-2096, 2020.

[14] CHEW W. C. Waves and fields in inhomogeneous media. IEEE press, 1995. 